\title{
Wakeful rest alleviates interference-based forgetting
}

\author{
Tom Mercer \\ University of Wolverhampton
}

Correspondence address:

Tom Mercer

Psychology Division

University of Wolverhampton

Wolverhampton, UK

WV1 1LY

Email: t.mercer2@wlv.ac.uk

Telephone: $+44(0) 1902321368$

Running head: Wakeful rest alleviates forgetting. 


\begin{abstract}
Retroactive interference - the disruptive influence of events occurring after the formation of a new memory - is one of the primary causes of forgetting. Placing individuals within an environment that postpones interference should therefore greatly reduce the likelihood of information being lost from memory. For example, a short period of wakeful rest should diminish interference-based forgetting. To test this hypothesis, participants took part in a foreign language learning activity and were shown English translations of 20 Icelandic words for immediate recall. Half of the participants were then given an 8 min rest before completing a similar or dissimilar interfering distractor task. The other half did not receive a rest until after the distractor task, at which point interference had already taken place. All participants were then asked to translate the Icelandic words for a second time. Results revealed that retention was significantly worse at the second recall test, but being allowed a brief rest before completing the distractor task helped reduce the amount of forgetting. Taking a short, passive break can shield new memories from retroactive interference and alleviate forgetting.
\end{abstract}




\section{KEY WORDS}

Forgetting, interference, memory, rest. 


\section{INTRODUCTION}

Human memory is notoriously unreliable and the amount of information that we forget can be surprising. For instance, there are plentiful cases of convictions arising from mistaken eyewitness identification (e.g. Pezdek, 2012), indicating a failure of memory, and events such as nearaccidents on the road appear to be forgotten with alarming speed (Chapman \& Underwood, 2000). Understanding the processes surrounding memory distortion is therefore extremely important and, unsurprisingly, there is a long history of research examining forgetting within experimental psychology (e.g. Britt, 1935; Ebbinghaus, 1885; Müller \& Pilzecker, 1900). Following decades of research, there is now some consensus amongst cognitive psychologists that information loss often arises through a process termed retroactive interference, or RI (see Hardt, Nader, \& Nadel, 2013, for discussion). RI occurs when the presentation of new information distorts or eradicates an existing representation and it is recognised as a potent cause of forgetting (Nairne \& Pandeirada, 2008).

As such, finding ways of reducing RI should lead to improvements in retention. One approach involves learning information within an "interference-free" context - if RI is absent, or even minimised, memories should have a greater likelihood of being recalled in the future. Sleep, for example, should create the perfect interference-free environment and would be expected to preserve memories in comparison to the waking state. The classic study of Jenkins and Dallenbach (1924) found that sleeping during the retention interval led to better recall than remaining awake, and more recent research indicates that even brief napping can have a beneficial effect. Lahl, Wispel, Willignes, and Pietrowsky (2008) asked participants to learn lists of 30 adjectives for a recall test that occurred an hour later. All volunteers were tested on two 
occasions: once when they were allowed to sleep during the 60 min interval (no RI) and once when they were asked to play computer games. Recall was higher when participants slept in comparison to when they stayed awake, and a second experiment revealed that a nap of just 6 mins led to a significant improvement in recall, in comparison to the no-sleep condition.

Research has also established that removing RI during wakeful states boosts retention. In a pioneering experiment, Müller \& Pilzecker (1900) documented that retention in an interferencefree condition was significantly better than a condition that incorporated some form of interference. Recent research continues to replicate this effect (e.g. Della Sala, Cowan, Beschin, \& Perini, 2005; Dewar, Cowan, \& Della Sala, 2007) and so retention might be similarly aided by a period of wakeful rest that occurs before RI. It has been proposed that delaying interference by employing a rest period allows the memory to become more resistant to subsequent interference (Wixted, 2004) and numerous studies published in the twentieth century explored this "temporal gradient" of RI. Most of these early studies employed a similar procedure, in which participants learnt a list of stimuli for a subsequent memory test. Prior to recall, they were asked to learn a second list (interpolated learning) that was designed to introduce interference. To explore the effects of the rest period, the temporal position of the interpolated learning could be varied (see Britt, 1935, for a review of the very first experiments).

Unfortunately, the evidence for a temporal gradient of RI and a beneficial effect of rest is mixed. Müller and Pilzecker (1900) showed participants lists of nonsense syllables (presented within pairs), followed by interpolated learning. After this, there was a paired associate recall test. Results confirmed that the temporal position of the second list was crucial - if individuals were asked to learn new information just $17 \mathrm{sec}$ after the first list, recall was impaired. But a gap of 6 mins was sufficient to protect memory of the first list (Lechner, Squire, \& Byrne, 1999, 
summarise this pioneering work). Subsequent studies supported Müller and Pilzecker's (1900) original findings (e.g. Landauer, 1974; Skaggs, 1925; Spencer, 1924), but other experiments found no effect of the temporal position of interference. For example, Robinson's (1920) participants learnt a list of numbers for a recall test that occurred after an interval of approximately 20 mins. In some conditions participants were required to learn a second list of numbers, but Robinson varied the position of this interpolated learning (it took place either immediately after original learning or following gaps of 5,10 , or 15 mins). The temporal placement of the interpolated learning was unimportant, and this has been confirmed by others (e.g. Archer \& Underwood, 1951; Houston, 1967). Furthermore, work by McGeoch (1933) discovered that interpolated learning had a more detrimental effect if it occurred immediately before recall (i.e. when interference is delayed), rather than directly after original learning (see Abra, 1969, and Newton \& Wickens, 1956, for analogous results). In a review of these findings, Wickelgren (1974) argued that the effect of RI is "independent of the interval separating original and interpolated learning” (p. 775). In essence, delaying RI does not alleviate forgetting.

There are, however, some serious methodological problems with these early experiments (Wixted, 2004). Numerous classic studies used long gaps between original learning and recall, sometimes requiring participants to leave the laboratory and return at a later date (Underwood, 1957). This is problematic since it is very difficult to control the degree of RI that occurs over these lengthy periods, particularly when participants are outside the laboratory. As such, the participant could be subjected to extensive amounts of RI during the interval before the formal interpolated learning activity. Similarly, researchers often failed to control RI occurring within the laboratory - Robinson (1920) gave participants reading material when they were not formally learning the second list, introducing a major confound. 
Wixted (2004) has outlined further problems with the studies that placed interpolated learning immediately before recall (e.g. McGeoch, 1933; Newton \& Wickens, 1956). By positioning interpolated learning directly prior to recall, researchers may have inadvertently introduced retrieval inhibition and consequently impaired participants' ability to recall the correct stimuli. Fortunately some classic experiments placed the interfering event in the middle of the retention interval (e.g. Sisson, 1939). In these cases, there was evidence for a temporal gradient of RI, with recall performance improving when the interpolated learning was placed half way into the retention interval, rather than at the beginning. Wixted has also re-analysed some of the older data that did not show temporal gradient effects and found that recall was better when interpolated learning was placed in the middle of the retention interval, rather than at the beginning or end.

More recent research has suggested that delaying interference through a period of wakeful rest does alleviate forgetting. Such studies have clearer controls and take care to minimise or eliminate external interference during the resting state. For example, Dewar, Alber, Butler, Cowan, and Della Sala (2012) used a within groups design and asked elderly adults to memorise a short story for immediate recall. After this, there was a 10 min delay. In one condition participants rested in a darkened room and in another condition they completed distracting spotthe-difference tasks. At this point, other spot-the-difference tasks occurred to ensure that some RI took place in the rest condition. Participants were then unexpectedly asked to recall the details of the story for a second time. A further recall test took place one week later. Impressively, individuals recalled more details when stories had been followed by a rest period prior to the spot-the-difference task and, strikingly, this improvement was maintained after a seven day period. 
Dewar, Alber et al.'s (2012) data suggest a brief, interference-free period of wakeful rest does protect memories from subsequent RI. This is a fascinating discovery since it offers a simple, effective technique for diminishing forgetting. However, there are three reasons to be cautious when interpreting these results. Firstly, the participants recruited by Dewar, Alber et al. were elderly ( $M=70+$ years old). Since there is some evidence that older adults show more rapid forgetting than younger adults (Wheeler, 2000) and may be more vulnerable to interference (e.g. Ebert \& Anderson, 2009), any beneficial effect of wakeful rest may be confined to those who are already quite susceptible to forgetting. Perhaps younger adults are capable of maintaining new memories without a resting period, even when interfering events are present.

Secondly, it is uncertain whether wakeful rest can protect against different types of RI. The distractor tasks used by Dewar, Alber et al. (2012) required participants to identify differences between pairs of pictures, hence the memory and distractor task were quite distinct. Whilst Dewar et al. (2007) have shown that any form of distractor task can disrupt recall, such dissimilar distractors are predicted to have a very mild effect on retention in neurologically intact humans (Dewar, Cowan, \& Della Sala, 2010). Indeed, some classic studies have determined that $\mathrm{RI}$ is more substantial and damaging when there is a degree of similarity between the interfering stimuli and to-be-memorised items (e.g. Harden, 1929; McGeoch, 1931; McGeoch \& McDonald, 1931; McGeoch \& McGeoch, 1936; Robinson, 1920). As such, it remains possible that wakeful rest can only negate the negative effects of weaker nonspecific interference (where memory and distractor tasks are very disparate). It is uncertain whether a rest period can protect newly formed memories from highly similar interfering items.

Thirdly, recent studies have not consistently reported a beneficial effect of wakeful rest prior to RI. Dewar, Fernandez Garcia, Cowan, and Della Sala (2009) assessed how delayed 
interference affected retention. Amnesic patients and 12 matched controls (mean age > 70) were asked to learn and immediately recall a word list. Then, after 9 mins, a second and unexpected recall test occurred, but the retention interval was either unfilled or contained an interfering distractor task (picture-naming). When the distractor task was present, it was either placed at the beginning (no rest), middle (3 min rest) or end ( 6 min rest) of the retention interval. As expected, amnesic patients showed a significant improvement in retention when the distractor task occurred at the end of the retention interval, rather than at the beginning, but the beneficial effect of rest was less evident for control participants. Indeed, the temporal position of the distractor task had no reliable effect. Additionally, performance following an unfilled interval was significantly better than in the two rest conditions. These data contradict Dewar, Alber et al. (2012) and imply that RI will disrupt memory, even if it is delayed by a rest period. This may be more evident when there is a degree of overlap between the memory and distractor tasks Dewar et al.'s (2009) picture-naming distractor included a verbal component which may have led to substantial RI that the rest period was incapable of diminishing.

In summary, whilst Dewar, Alber et al. (2012) found that resting had a prolonged positive influence on retention, Dewar et al.'s (2009) data showed that brief phases of rest did not preserve retention rates in participants with intact memory. Studies investigating the temporal gradient of RI during the early twentieth century were mixed, and other recent literature examining wakeful rest and minimal RI has primarily focussed on patients with amnesia and other forms of cognitive impairment (e.g. Cowan, Beschin, \& Della Sala, 2004; Dewar et al., 2009; Dewar, Della Sala, Beschin, \& Cowan, 2010; Dewar, Pesallaccia, Cowan, Provinciali, \& Della Sala, 2012). Whilst this research has yielded key insights into how interference operates in 
patients suffering from cognitive impairment, it is less obvious how neurologically intact individuals are affected by periods of wakeful rest. Further work is necessary.

The present experiment was designed to examine the impact of wakeful rest on recall. Although past research has consistently shown that retention in an interference-free condition will exceed retention when RI is present, it remains uncertain whether postponing RI will yield a positive outcome. This study extended issues addressed in previous work, particularly the research of Dewar and colleagues, and focussed on two open questions. Firstly, the generalizability of the wakeful rest effect was investigated by recruiting a sample of younger adults. This is necessary since recent work has primarily been confined to elderly adults (especially those with cognitive impairments) and classic studies that employed younger samples led to mixed results. Secondly, the present experiment intended to subject participants to more substantial RI (at least in one condition) and assess whether a period of rest was still valuable.

Individuals took part in a foreign language learning exercise and were asked to memorise the English translation of 20 Icelandic words. After the first recall test, half of the individuals were given an 8 min rest period (immediate rest) and the other half underwent a distractor task (delayed rest). Participants in the latter condition did not receive a rest until they had completed the distractor task, at which point RI should have already occurred. To assess whether the type of distractor material had an effect, similar and dissimilar distractor tasks were created. The former involved the presentation and subsequent recognition of Norwegian-English word pairs (predicted to cause similarity-based interference), whereas the latter employed a facial recognition procedure (a dissimilar interference task). Importantly, both distractor tasks required participants to form new memories, but the degree to which the distractor material overlapped 
with the Icelandic-English pairs greatly differed. After completing the distractor task, participants were given a second and unexpected Icelandic recall test.

If the rest period really helps protect new memories from interference, there should be significantly less forgetting over the retention interval in the immediate rest condition, in comparison with delayed rest. A similarity-based view of RI would expect the NorwegianEnglish distractor task to cause more disruption than the face stimuli, although whether a rest period will be capable of minimising this effect is uncertain.

\section{METHOD}

\section{Participants}

87 participants ( 74 females, 13 males) aged between 18 and $51(M=21.28, S D=6.21)$ were recruited for the experiment. The majority of participants were young adults, aged between 18 and 25 , and only 10 individuals were aged 26 or older. Age had no effect on recall performance. Participants were first year undergraduate psychology students from the University of Wolverhampton who took part during a research methods practical session.

\section{Materials}

Highly imageable English words (500-599 imageability score) between four and seven letters in length were selected from the Bristol Norms database (Stadthagen-Gonzalez \& Davis, 2006). Norwegian and Icelandic translations were then provided for each English word by native 
Norwegian and Icelandic speakers. These Scandinavian languages were chosen because of their unfamiliarity to most English speakers and the final word pairs were selected on the basis of two criteria: the Norwegian/Icelandic words had to consist of four to nine letters and they had to bear no resemblance to the English translation. 20 Icelandic-English and 42 Norwegian-English pairs were generated for the experiment. The Norwegian-English pairings were used for the similar distractor task and 30 of them were adapted for the recognition test.

Stimuli for the dissimilar distractor task were selected from the Tarrlab Face Database (stimulus images courtesy of Michael J. Tarr, Center for the Neural Basis of Cognition, Carnegie Mellon University, http://www.tarrlab.org/). This contains photos of over 200 individuals, of which 84 were selected for this experiment. Photos were pseudo-randomly selected with the criteria that individuals had a neutral expression and were facing the camera. Individuals wearing glasses were excluded. The final face pairs depicted photos of two individuals of the same race and sex. Each image was shown in colour (dimensions: 250x250; resolution: $72 \mathrm{dpi}$ ).

Recall sheets featured all 20 Icelandic words arranged in a random order. In addition, a demographic questionnaire was created and included questions about participants' age, sex, and first and second language abilities. Stimuli were presented on a PC running Cedrus SuperLab software (version 4.5). The stimuli were displayed via a projector and six Samsung HD 37" LED TVs suspended from the ceiling. All textual stimuli were presented in the centre of the screen (Tahoma size 72).

\section{Design and Procedure}


The experiment employed a 2 (recall time: immediate vs. delayed) x 2 (rest period: immediate vs. delayed) x 2 (distractor task: similar vs. dissimilar) mixed design. There were four independent conditions based upon the rest period and distractor task: "immediate rest, similar distractor task", "immediate rest, dissimilar distractor task", "delayed rest, similar distractor task" and "delayed rest, dissimilar distractor task". Participants were randomly allocated to one of four conditions and individuals were tested within groups.

The individuals were seated within a large room with approximately $170 \mathrm{~cm}$ between each person. They were told that they would complete separate recall and recognition tests. After reading the information sheet and signing the consent form, participants were given an experimental booklet and told to refrain from turning the page until instructed. At this point they were asked to go to the second page and complete the demographics questionnaire. The 20 Icelandic-English word pairs were then shown via the projector and six screens. The pairs were preceded by a fixation cross $(100 \mathrm{~ms})$ and remained on screen for $5 \mathrm{~s}$. The next pair was displayed after an interval of $3 \mathrm{~s}$. After the final word pair was displayed, participants were told to turn to the fourth page of their booklet and were given 3 mins to provide the English translation for as many Icelandic words as possible (immediate recall).

Participants in the immediate rest condition were then given an 8 min rest before the distractor task. The lights were dimmed and participants were asked to rest quietly (see Dewar, Alber et al., 2012). They were encouraged to close their eyes and place their head on the desk. After 8 mins had elapsed, participants proceeded to the distractor task. Individuals subjected to similar interference were shown 42 Norwegian-English word pairs whereas those in the dissimilar interference condition were shown 42 pairs of human faces of the same race and sex. Each trial was preceded by a fixation cross $(100 \mathrm{~ms})$ and stimuli remained on screen for $5 \mathrm{~s}$. The next trial 
commenced after a $3 \mathrm{~s}$ interval. After all stimuli had been presented, participants turned to the final page of their experimental booklet for the recognition test. They were shown 30 pairs on screen, each for $5 \mathrm{~s}$, and required to determine whether it was accurate ("Yes" trial) or inaccurate ("No" trial). Fifteen pairs accurately presented a Norwegian word with the correct translation, or two faces that had previously been paired, whereas the other 15 were erroneous. Successive stimulus pairs were shown after blank intervals lasting $5 \mathrm{~s}$ and the entire distractor task was approximately 11 mins in length. Participants in the delayed rest condition were asked to begin the distractor task directly after the recall test. They were not given the rest until the distractor task had been completed, at which point RI should have already occurred. Crucially, the length of the interval between immediate and delayed recall was identical in all conditions.

\section{"Figure 1 about here"}

After the rest and distractor task, the experimental booklets were collected and participants were given another recall test (see Figure 1). The procedure for this delayed recall test was identical to the previous one, and the Icelandic words were again arranged randomly. To reduce the likelihood of participants actively rehearsing the material during the rest period, the second recall test was unexpected. It was therefore unlikely that individuals were attempting to maintain the Icelandic-English pairs through on-going rehearsal. In addition, given the novel and unfamiliar nature of Icelandic words (e.g. "vængur"), coupled with the requirement that participants memorise the English translation associated with specific Icelandic words, an effective rehearsal strategy may have been quite difficult. 


\section{RESULTS}

Three individuals who misunderstood the experimental instructions were excluded from the data analysis. Eleven participants did not speak English as a first language, but they were highly proficient in English and there was no difference in recall performance between native and nonnative speakers. Since removing these individuals did not affect the outcome of the analyses, they were retained in the sample ${ }^{1}$. No participant was able to speak Icelandic or Norwegian.

\section{"Table 1 about here"}

Table 1 shows the proportion of correct English translations for both recall periods in the four different conditions. When examining the effect of rest, there appeared to be less forgetting when participants were given an immediate rest (i.e. when RI was postponed), in comparison with delayed rest conditions. There also appeared to be no difference between the similar and dissimilar distractor tasks in the immediate rest conditions (both led to an identical drop in performance), although this pattern changed in the delayed rest condition. Here there seemed to be less forgetting when the distractor task was dissimilar to the Icelandic-English pairs (recall declined by .04, from .3 to .26), rather than similar (recall declined by .07, from .39 to .32 ). Still, this difference is modest.

\footnotetext{
${ }^{1}$ Group sizes were as follows: Delayed rest, similar distractor task, $N=21$; delayed rest, dissimilar distractor task, $N=18$, immediate rest, similar distractor task, $N=21$, immediate rest, dissimilar distractor task, $N=24$. Despite some differences in the sample sizes within the conditions, a nonparametric equivalent of Levene's test suggested that the homogeneity of variance assumption had been met (Nordstokke \& Zumbo, 2010).
} 
"Figure 2 about here"

To analyse these data, a 2 (recall time: immediate vs. delayed) x 2 (rest period: immediate vs. delayed) x 2 (distractor task: similar vs. dissimilar) mixed ANOVA was conducted. The analysis revealed a significant main effect of recall time, with immediate recall being higher than delayed recall, $F(1,80)=35.07, M S E=.002, p<0.0005$, partial $\eta^{2}=.31$, so forgetting had occurred. There was no main effect of rest period, $F(1,80)=.48, M S E=.078$, or distractor task, $F(1,80)=$ $.65, M S E=.078$, but there was a significant interaction between recall time and rest period, $F(1$, $80)=4.71, M S E=.002, p=.033$, partial $\eta^{2}=.06$. Figure 2 displays this interaction. Simple effects analysis suggested that there was no difference in retention between rest conditions at immediate recall $(p=.527)$, but there was significantly better performance following an immediate (rather than delayed) rest during the second recall test $(p=.026)$. For further exploration, delayed recall scores were subtracted from immediate recall to generate a simple measure of forgetting. An independent $t$ test indicated that mean forgetting in the delayed rest condition $(M=.05, S D=.07)$ was significantly higher than in the immediate rest condition $(M=$ $.02, S D=.05), t(82)=2.26, p=.013$ (one-tailed), $d=.49$. Hence there was more forgetting when participants did not receive a rest before RI. Conversely, the interaction between recall time and distractor task was non-significant, $F(1,80)=.98, M S E=.002$. Figure 3 suggests that both the similar and dissimilar distractor tasks led to equivalent forgetting over the retention interval. All other interactions were non-significant.

"Figure 3 about here" 


\section{DISCUSSION}

The present experiment aimed to examine whether a brief period of wakeful rest could reduce forgetting. Participants learnt Icelandic-English word pairs for immediate recall and were then subjected to a distractor task that either involved learning and recognising Norwegian-English word pairs (material very similar to the Icelandic-English stimuli) or learning and recognising pairs of human faces (materials very dissimilar to the Icelandic-English stimuli). Crucially, however, some participants were given an 8 min rest directly after immediate recall, whereas others did not receive a rest until after completing the distractor task, by which point they had already been subjected to RI.

The results suggested that receiving an immediate rest did help alleviate forgetting in comparison with participants who were given a delayed rest. Placing individuals within an environment that postpones RI does seem to have a positive effect on retention. These findings support Dewar, Alber et al. (2012), who found a beneficial effect of wakeful rest in normally aging adults, along with some of the earliest experiments examining the temporal gradient of RI (Landauer, 1974; Müller \& Pilzecker, 1900; Skaggs, 1925; Spencer, 1924). Given Dewar, Alber et al.'s sample, there was concern that rest might only help those who are already more vulnerable to forgetting, but the present data dispel this suggestion and show that wakeful rest lessens forgetting in younger adults too. Indeed, the magnitude of this effect is worth noting. Cowan, Saults, and Nugent (2001) devised an algorithm that permits the maximum possible amount of forgetting to be computed. Applying this algorithm to the present data, it was revealed that individuals in the delayed rest condition forgot $15.57 \%$ of the maximum possible amount. 
This dropped to $6.83 \%$ in the immediate rest condition - a difference of $8.74 \%$. Wakeful rest protects new memories from RI.

In contrast to the present findings, Dewar et al. (2009) did not find any substantial positive effect of wakeful rest in their control participants. These individuals were either directly subjected to a distractor task or were given a 3 or 6 min rest. After the rest, participants completed the distractor task, although in a fourth condition the entire retention interval was unfilled. Performance was best when the distractor task was absent, whereas there was no significant difference between no-rest, $3 \mathrm{~min}$ and 6 min rest conditions. It is uncertain why Dewar et al. (2009) did not find an advantageous effect of wakeful rest for these individuals, but a lack of power is one possibility. Retention following a 6 min gap was slightly, albeit nonsignificantly, better than no-rest. Alternatively, the rest periods could have been too short. The present experiment used an 8 min period, whereas Dewar, Alber et al. (2012) employed a 10 min rest. Perhaps 3 and 6 mins are simply not long enough to significantly improve retention (but see Müller \& Pilzecker, 1900), and determining whether there is a critical rest period duration would be an interesting topic for future research.

Nevertheless, 8 mins of wakeful rest is sufficient to shield new memories from RI. It is unlikely that this effect resulted from participants employing an intentional rehearsal strategy over the rest period for two major reasons. Firstly, the word-pair stimuli were inherently difficult to rehearse given that participants had to remember the correct word combination, and the Icelandic words were novel and unfamiliar. Secondly, and more importantly, the delayed recall test was unexpected. Given the surprising nature of the second recall, it is doubtful that participants spent their time rehearsing. An alternative possibility is that wakeful rest allows memories to consolidate in the absence of interference. Such uninterrupted consolidation would 
presumably permit the representation to be stabilised and become more resilient. Dewar, Alber et al. (2012) favoured this explanation and it could be applied to the present findings too. However, rather than rest permitting consolidation, it is equally possible that rest periods prevent memories from being confused. Temporal distinctiveness models such as Brown, Neath, and Chater's (2007) SIMPLE predict that memory retrieval is determined by the temporal proximity of different memory traces. A representation that is temporally isolated from its neighbours will be more distinct than representations that were learnt within a temporally crowded context. This account is readily applicable to the present experiment. Specifically, in the immediate rest condition, Icelandic-English pairs were temporally separated from the distractor task and so memory for these stimuli should have been more distinct. In the delayed rest condition there was greater temporal crowding and consequently more forgetting. Importantly, this notion of temporal distinctiveness can explain other findings that have traditionally been linked to consolidation. For example, Lewandowsky, Ecker, Farrell, and Brown (2012) show that the temporal gradient of RI is fully explicable by SIMPLE, without the need to invoke a consolidation process (see also Ecker and Lewandowsky, 2012, for discussion of the weaknesses of consolidation theory).

The present experiment cannot disentangle consolidation and distinctiveness-based accounts since both models predict that postponing RI will reduce forgetting. Future behavioural studies will need an ingenious design to fully extricate the predictions of these two accounts, and there may be other plausible explanations too. Yet despite uncertainty regarding the best theoretical account, there may be some useful applications from the present findings. Specifically, short periods of wakeful rest could be used as a simple technique to aid recall. It is already known that wakeful rest boosts retention in those suffering from different forms of cognitive impairment 
(Dewar, Della Sala, et al., 2010; Dewar, Pesallaccia, et al., 2012) but, since it can also help those with intact memory, it may prove useful within educational settings where students need to remember information for subsequent tests. Similarly, it would be intriguing to see whether a rest period can aid retention of emotional events, e.g. a crime.

In addition to the impact of rest, the type of distractor task seemed to be inconsequential. Learning material similar to the Icelandic-English pairs did not lead to greater forgetting when compared with the effect of dissimilar material. This seems very surprising since some classic studies found that interference is driven by the similarity of the original and interpolated learning (e.g. Harden, 1929; McGeoch, 1931; McGeoch \& McGeoch, 1936). One possible explanation for the absence of an effect of similarity is a lack of power. The values in Table 1 do show that the similar distractor task led to more forgetting than the dissimilar distractor task in the delayed rest condition, although this difference appeared to vanish in the immediate rest condition. To investigate the magnitude of this effect, Cohen's $d$ was calculated from the amount of observed forgetting (this is computed by subtracting delayed recall from immediate recall). Within the immediate rest condition, the effect size for distractor task was tiny $(d=.066)$, lending further support to the suggestion that the content of the distractor task was irrelevant. The distractor task effect size within the delayed rest condition was higher $(d=.32)$, although still small. These data indicate that participants immediately subjected to the similar distractor task showed more forgetting than those asked to complete a dissimilar distractor task, but this was a relatively small effect and did not reach statistical significance.

Whilst this seems to contradict previous findings, the effect of similarity on RI is more complex than might be expected. Logically, interference should become more destructive as the similarity between original and interpolated learning increases, but this is not always the case. 
For example, many classic interference studies required participants to learn a list of word pairs (list 1), with each pair consisting of a stimulus word and a response word. This was typically followed by an interpolated learning phase, involving the retention of a new list of word pairs (list 2). To heighten interference during list 2 learning, participants could be given the same stimulus words from list 1, but new response words. However, if the response words on list 2 are similar to the response words on list 1, RI disappears (see Anderson, Green, \& McCulloch, 2000). Osgood (1949) termed this effect the "similarity paradox" and it usefully highlights that greater similarity does not always lead to more forgetting. Conversely, interfering material that is dissimilar to the original memory may be quite capable of disrupting recall. Some of the earliest studies discovered that it is the presence of a distractor task that is crucial, rather than its content (e.g. Müller \& Pilzecker, 1900), and Wixted (2004) has proposed that any interfering event can cause the loss of pre-consolidated memories. In support of this, Dewar et al. (2007) reported that a range of distractor tasks impair recall in comparison with a no-interference control condition. They asked participants to remember a list of nouns and, during an $8 \mathrm{~min}$ retention interval, participants were subjected to different types of distraction in different conditions. These included a verbal memory task, visual imagery tasks, mathematical problems and a tone detection procedure. Individuals in the control condition were not subjected to a distractor task. Recall scores were higher when RI was absent than when a distractor task was present, but, crucially, there were no significant differences in retention according to the type of distractor task - undertaking a tone detection task was as detrimental to word recall as completing an additional verbal memory task.

Advocates of consolidation theory might argue that the present results document that any distractor task leads to forgetting by stopping consolidation, but this is not necessarily the case. 
Whilst the material presented during the distractor tasks may have interfered with the memory of the Icelandic-English word pairs, this does not mean that the distractor task prevented or impaired consolidation. Yet the present findings do raise some interesting questions regarding the nature of RI and the mechanism through which it operates. Perhaps the difficulty of the distractor task is pertinent. During the recognition test, participants performed significantly poorer on the dissimilar $(M=.60)$ than similar $(M=.75)$ distractor task $(p<.0005)$, so memorising and recognising face pairs seemed to be more challenging than learning NorwegianEnglish word combinations. A more difficult task might have higher cognitive demands; hence distractor task difficulty could potentially equalise the damaging effect of distractor task similarity. This would reduce the difference that might otherwise have been expected between the two types of distractor task used here. Alternatively, since both distractor tasks required the formation of new memories, it could be the act of creating memories that leads to RI, regardless of what is being learnt. This is a tentative suggestion because the present experiment did not include a condition that lacked a distractor task (the primary purpose of this study was to examine whether varying the position of a distractor task affected the rate of forgetting). Researchers wishing to further examine the effects of different types of distractor task on wakeful rest would need to incorporate a condition that removed external distraction to yield a more definitive answer.

\section{CONCLUSION}


Individuals wishing to memorise new material should give themselves a short rest. During this time they need only relax, ensuring any distractions are kept to a minimum. This may help alleviate forgetting that would otherwise be caused by interference.

Word count (Introduction, Method, Results, Discussion): 5,647

Word count (References): 1,152 


\section{ACKNOWLEDGEMENTS}

This work was supported by an Early Researcher Award Scheme (ERAS) grant from the University of Wolverhampton. I would like to thank Dr Marie Poirier and two anonymous reviewers for helpful comments on an earlier version of this manuscript. I am also grateful to Louise Davies, Ian Jukes and Phil Oates for their technical support, Julie Jebsen for her help in creating the Norwegian-English word pairs and coding the data, and Hannah Anderson for her assistance in creating the Icelandic-English word pairs. The face images used in this experiment were provided courtesy of Michael J. Tarr, Center for the Neural Basis of Cognition, Carnegie Mellon University, http://www.tarrlab.org/. 


\section{REFERENCES}

Abra, J. C. (1969). List-1 unlearning and recovery as a function of the point of interpolated learning. Journal of Verbal Learning and Verbal Behavior, 8, 494-500. doi: 10.1016/S00225371(69)80093-9

Anderson, M. C., Green, C., \& McCulloch, K. C. (2000). Similarity and inhibition in long-term memory: Evidence for a two-factor theory. Journal of Experimental Psychology: Learning, Memory, and Cognition, 26, 1141-1159. doi: 10.1037//0278-7393.26.5.1141

Archer, J. E. \& Underwood, B. J. (1951). Retroactive inhibition of verbal associations as a multiple function of temporal point of interpolation and degree of interpolated learning. Journal of Experimental Psychology, 42, 283-290. doi: 10.1037/h0059924

Britt, S. H. (1935). Retroactive inhibition: A review of the literature. Psychological Bulletin, 32, 381-440. doi: $10.1037 / \mathrm{h} 0061316$

Brown, G. D. A., Neath, I., \& Chater, N. (2007). A temporal ratio model of memory. Psychological Review, 114, 539-576. doi: 10.1037/0033-295X.114.3.539

Chapman, P., \& Underwood, G. (2000). Forgetting near-accidents: the roles of severity, culpability and experience in the poor recall of dangerous driving situations. Applied Cognitive Psychology, 14, 31-44. doi: 10.1002/(SICI)1099-0720(200001)14:1<31::AIDACP622>3.0.CO;2-9

Cowan, N., Beschin, N., \& Della Sala, S. (2004). Verbal recall in amnesiacs under conditions of diminished retroactive interference. Brain, 127, 825-834. doi: 10.1093/brain/awh107

Cowan, N., Saults, J. S., \& Nugent, L. D. (2001). The ravages of absolute and relative amounts of time on memory. In H. L. Roediger III, J. S. Nairne, I. Neath, \& A. Suprenant (Eds.), The 
nature of remembering: Essays in honor of Robert G. Crowder (pp. 315-330). Washington, D.C: American Psychological Association.

Della Sala, S., Cowan, N., Beschin, N., \& Perini, M. (2005). Just lying there, remembering: Improving recall of prose in amnesic patients with mild cognitive impairment by minimising interference. Memory, 13, 435-440. doi: 10.1080/09658210344000387

Dewar, M., Alber, J., Butler, C., Cowan, N., \& Della Sala, S. (2012). Brief wakeful resting boosts new memories over the long term. Psychological Science, 23, 955-960. doi: $10.1177 / 0956797612441220$

Dewar, M. T., Cowan, N., \& Della Sala, S. (2007). Forgetting due to retroactive interference: A fusion of Muller and Pilzecker's (1900) early insights into everyday forgetting and recent research on anterograde amnesia. Cortex, 43, 616-634. doi: 10.1016/S0010-9452(08)70492-1

Dewar, M., Cowan, N., \& Della Sala, S. (2010). Forgetting due to retroactive interference in anterograde amnesia. In S. Della Sala (Ed.), Forgetting (pp. 185-209). Hove, UK: Psychology Press.

Dewar, M., Della Sala, S., Beschin, N., \& Cowan, N. (2010). Profound retroactive interference in anterograde amnesia: What interferes? Neuropsychology, 24, 357-367. doi: 10.1037/a0018207

Dewar, M., Fernandez Garcia, Y., Cowan, N., \& Della Sala, S. (2009). Delaying interference enhances memory consolidation in amnesic patients. Neuropsychology, 23, 627-634. doi: $10.1037 / \mathrm{a} 0015568$

Dewar, M., Pesallaccia, M., Cowan, N., Provinciali, L., \& Della Sala, S. (2012). Insights into spared memory capacity in amnestic MCI and Alzheimer's Disease via minimal interference. Brain and Cognition, 78, 188-199. doi: 10.1016/j.bandc.2011.12.005

Ebbinghaus, H. (1885). Uber das Gedächtnis. Leipzig: Dunker \& Humbolt. 
Ebert, P. L., \& Anderson, N. D. (2009). Proactive and retroactive interference in young adults, healthy older adults, and older adults with amnesic mild cognitive impairment. Journal of the International Neuropsychological Society, 15, 83-93. doi: 10.1017/S1355617708090115

Ecker, U. K. H., \& Lewandowsky, S. (2012). Computational constraints in cognitive theories of forgetting. Frontiers in Psychology, 3, 1-5. doi: 10.3389/fpsyg.2012.00400

Harden, L. M. (1929). A quantitative study of the similarity factor in retroactive inhibition. Journal of General Psychology, 2, 421-432. doi: 10.1080/00221309.1929.9918081

Hardt, O., Nader, K., \& Nadel, L. (2013). Decay happens: the role of active forgetting in memory. Trends in Cognitive Sciences, 17, 111-120. doi: 10.1016/j.tics.2013.01.001

Houston, J. P. (1967). Retroactive inhibition and point of interpolation. Journal of Verbal Learning and Verbal Behavior, 6, 84-88. doi: 10.1016/S0022-5371(67)80054-9

Jenkins, J. G., \& Dallenbach, K. M. (1924). Oblivescence during sleep and waking. American Journal of Psychology, 35, 605-612.

Lahl, O., Wispel, C., Willigens, B., \& Pietrowsky, R. (2008). An ultra short episode of sleep is sufficient to promote declarative memory performance. Journal of Sleep Research, 17, 3-10. doi: $10.1111 / \mathrm{j} .1365-2869.2008 .00622 . \mathrm{x}$

Landauer, T. K. (1974). Consolidation in human memory: Retrograde amnestic effects of confusable items in paired-associate learning. Journal of Verbal Learning and Verbal Behavior, 13, 45-53. doi: 10.1016/S0022-5371(74)80029-0

Lechner, H. A., Squire, L. R., \& Byrne, J. H. (1999). 100 years of consolidation - Remembering Müller and Pilzecker. Learning \& Memory, 6, 77-87. doi: 10.1101/lm.6.2.77 
Lewandowsky, S., Ecker, U. K. H., Farrell, S., \& Brown, G. D. A. (2012). Models of cognition and constraint from neuroscience: A case study involving consolidation. Australian Journal of Psychology, 64, 37-45. doi: 10.1111/j.1742-9536.2011.00042.x

McGeoch, J. A. (1931). The influence of four different interpolated activities upon retention. Journal of Experimental Psychology, 14, 400-413. doi: 10.1037/h0074435

McGeoch, J. A. (1933). Studies in retroactive inhibition: II. Relationships between temporal point of interpolation, length of interval, and amount of retroactive inhibition. Journal of General Psychology, 9, 44-57. doi: 10.1080/00221309.1933.9920912

McGeoch, J. A., \& McDonald, W. T. (1931). Meaningful relation and retroactive inhibition. American Journal of Psychology, 43, 579-588. doi: 10.2307/1415159

McGeoch, J. A., \& McGeoch, G. O. (1936). Studies in retroactive inhibition: VI. The influence of the relative serial positions of interpolated synonyms. Journal of Experimental Psychology, 19, 1-23. doi: 10.1037/h0060051

Müller, G. E., \& Pilzecker, A. (1900). Experimentelle Beiträge zur Lehre vom Gedächtniss. Zeitschrift für Psychologie, 1, 1-300.

Nairne, J. S., \& Pandeirada, J. N. S. (2008). Forgetting. In H. L. Roediger, III. (Ed.), Cognitive psychology of memory. Vol. 2. Learning and Memory: A Comprehensive Reference (pp. 179194). Oxford: Elsevier.

Newton, J. M., \& Wickens, D. D. (1956). Retroactive inhibition as a function of the temporal position of the interpolated learning. Journal of Experimental Psychology, 51, 149-154. doi: $10.1037 / \mathrm{h} 0043572$

Nordstokke, D. W., \& Zumbo, B. D. (2010). A new nonparametric Levene test for equal variances. Psicologica, 31, 401-430. 
Osgood, C. E. (1949). The similarity paradox in human learning: A resolution. Psychological Review, 56, 132-143. doi: 10.1037/h0057488

Pezdek, K. (2012). Fallible eyewitness memory and identification. In B. L. Cutler (Ed), Conviction of the innocent: Lessons from psychological research (pp. 105-124). Washington, DC, US: American Psychological Association.

Robinson, E. S. (1920). Studies from the psychological laboratory of the University of Chicago: Some factors determining the degree of retroactive inhibition. Psychological Monographs, 28, 1-57. doi: $10.1037 / \mathrm{h} 0093155$

Sisson, E. D. (1939). Retroactive inhibition: The temporal position of interpolated activity. Journal of Experimental Psychology, 25, 228-233. doi: 10.1037/h0055178

Skaggs, E. B. (1925). Further studies in retroactive inhibition. Psychological Monographs, 34, 160.

Spencer, L. T. (1924). A note regarding retroactive inhibition after a twenty-minute interval. American Journal of Psychology, 35, 466-467.

Stadthagen-Gonzalez, H., \& Davis, C. J. (2006). The Bristol norms for age of acquisition, imageability, and familiarity. Behavior Research Methods, 38, 598-605. doi: 10.3758/BF03193891

Underwood, B. J. (1957). Interference and forgetting. Psychological Review, 64, 49-60.

Wheeler, M. A. (2000). A comparison of forgetting rates in older and younger adults. Aging, Neuropsychology, and Cognition, 7, 179-193. doi: 10.1076/1382-5585(200009)7:3;1Q;FT179

Wickelgren, W. A. (1974). Single-trace fragility theory of memory dynamics. Memory \& Cognition, 2, 775-780. doi: 10.3758/BF03198154 
Wixted, J. T. (2004). The psychology and neuropsychology of forgetting. Annual Review of Psychology, 55, 235-269. doi: 10.1146/annurev.psych.55.090902.141555 


\section{TABLES}

Table 1

Mean (and standard deviation) for immediate and delayed recall according to rest period and type of distractor task

Immediate Rest

Distractor Task
Immediate recall
Delayed Rest
Similar

$.35(.20)$

$.36(.22)$
Dissimilar
$.33(.20)$

$.39(.19)$

$.30(.18)$
$.34(.22)$
Delayed recall
Immediate recall

.




\section{FIGURE CAPTIONS}

Figure 1. Diagrammatic representation of the experimental arrangement.

Figure 2. Mean proportion of English translations correctly recalled in the delayed and immediate rest conditions. Error bars indicate standard error of the mean.

Figure 3. Mean proportion of English translations correctly recalled in the similar and dissimilar distractor task conditions. Error bars indicate standard error of the mean. 
Figure 1.

Immediate rest

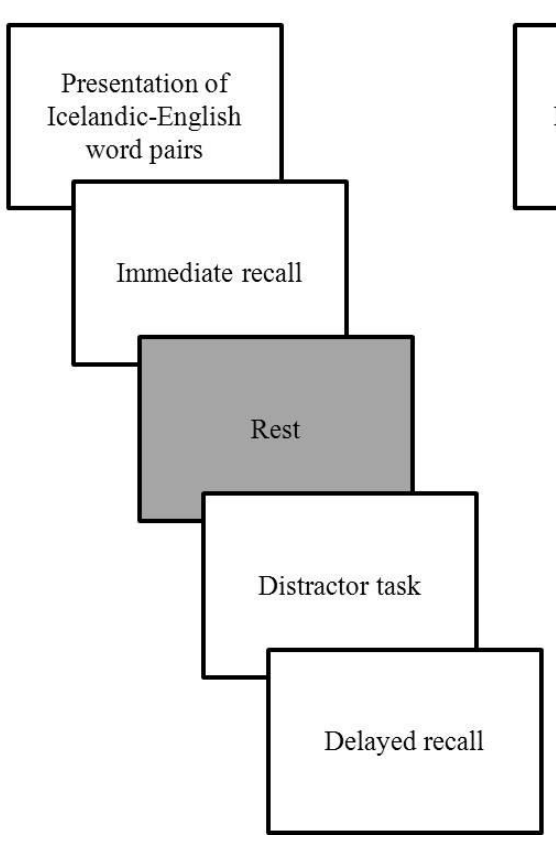

Delayed rest

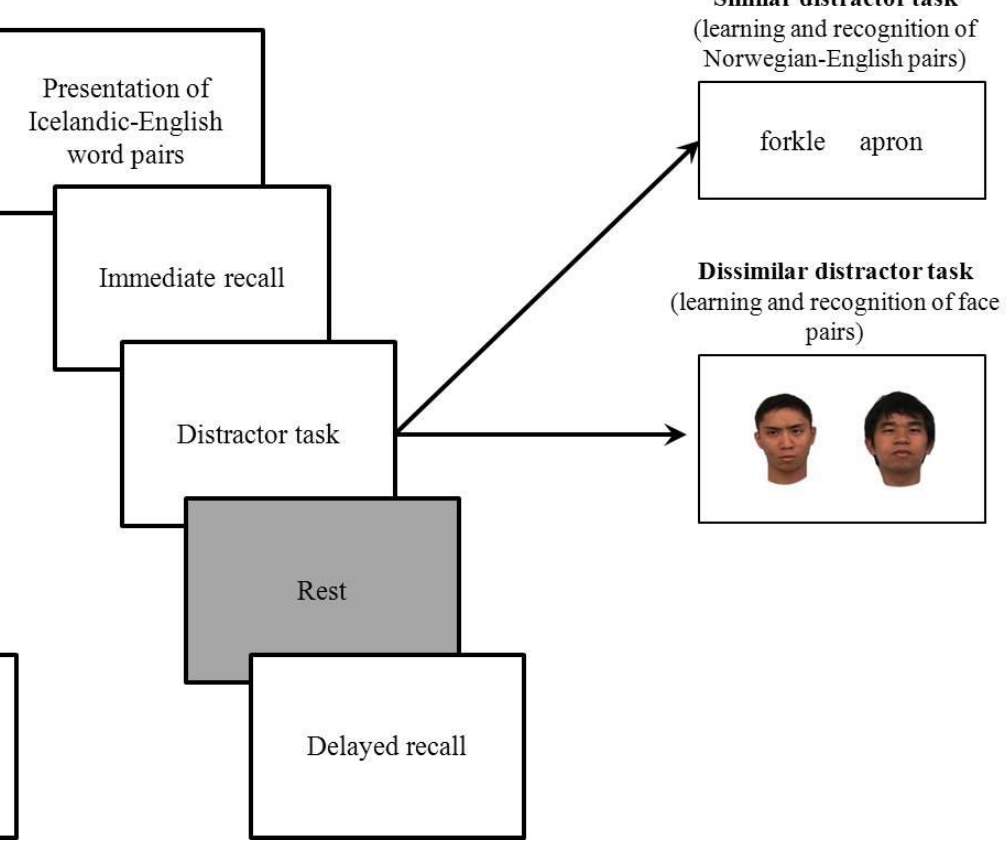


Figure 2.

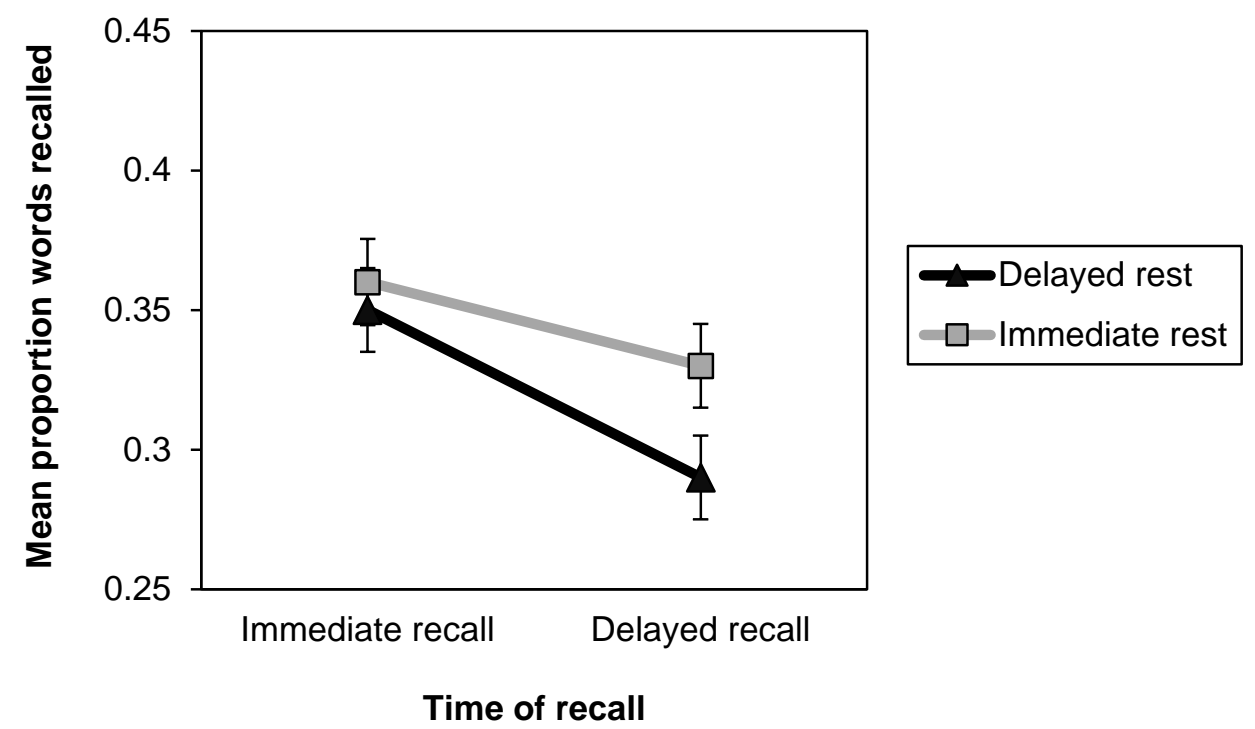


Figure 3.

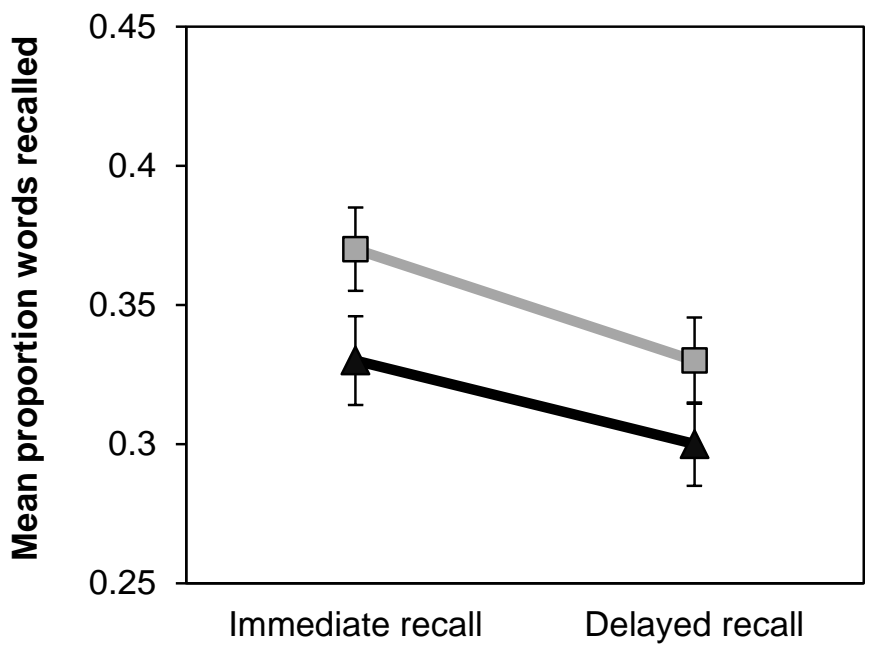

Dissimilar distractor task (faces)

$\square$ Similar distractor task (Norwegian)

Time of recall 